I. DIMITRIĆ

KODAI MATH. J.

14 (1991), 281-295

\title{
1-TYPE SUBMANIFOLDS OF THE COMPLEX PROJECTIVE SPACE
}

\author{
By IVko Dimitrić
}

\section{§ 0. Introduction.}

The standard way to define the complex projective space $C P^{m}$ is by means of Hopf fibration $\pi: S^{2 m+1} \rightarrow C P^{m}$ where $C P^{m}$ is obtained as a quotient space of $S^{2 m+1} \subset C^{m+1}$ under the natural action of the group $S^{1}$ of complex numbers of norm 1. It is not completely trivial to find an isometric embedding of $C P^{m}$ into a Euclidean space, the most natural one (the first standard embedding) defined as follows. For $p \in C P^{m}$ pick $z \in \pi^{-1}(p) \subset S^{2 m+1}$ and let $\phi(p)=z \bar{z}^{t}$ ( $z$ is regarded as a column vector in $C^{m+1}$ ). Then $\phi$ is a well defined map that embeds $C P^{m}$ into the set $H(m+1)$ of Hermitian matrices of degree $m+1$, the latter being a Euclidean space of dimension $N=(m+1)^{2}$. Now if $x: M^{n} \rightarrow \boldsymbol{C} P^{m}$ is an isometric immersion of a compact $n$-dimensional Riemannian manifold into the complex projective space, than we also have the associated immersion $\tilde{x}=\phi \circ x: M^{n} \rightarrow H(m+1)=E^{N}$.

On the other hand, there is a notion of finite type immersion $f: M^{n} \rightarrow E^{N}$ whereby a compact Riemannian manifold $M^{n}$ is said to be of $k$-type (via $f$ ) if $f$ is globally decomposable as $f=f_{0}+f_{1}+\cdots+f_{k}$, where $f_{0}=$ const (could be 0 ) and $f_{i}$ 's are vector eigenfunctions of Laplacian from $k+1$ different eigenspaces [3]. For example, a well known result of Takahashi [15] characterizes compact 1-type submanifolds as minimal in a hypersphere of $E^{N}$. Studying finite type immersions is difficult in general, but there have been several results on low type submanifolds of $C P^{m}$. In particular, A. Ros has the following classification of $C R$-minimal submanifolds of $C P^{m}$ which are of 1-type via the first standard embedding.

THEOREM A [10]. Let $M^{n}$ be a compact CR-minimal submanifold of $\boldsymbol{C} P^{m}$. Then $M^{n}$ is of 1-type via $\phi$ if and only if

a) $n$ is even and $M^{n}$ is congruent to the complex projective space $C P^{n / 2}$ immersed as a totally geodesic complex submanifold of $\boldsymbol{C} P^{m}$.

b) $M^{n}$ is a totally real minimal submanifold of a complex totally geodesic $\boldsymbol{C} P^{n}$ in $\boldsymbol{C} P^{m}$.

Received July 4, 1990; revised December 10, 1990. 
Ros' proof relies on the equivariancy of the embedding $\phi$ and uses both assumptions $C R$ and minimal in an essential way. In this paper, using somewhat different and more refined approach, we give an extension of this result (Th. 1). It turns out that one can classify 1-type submanifolds of $C P^{m}$ assuming them to be only $C R$ (or with parallel mean curvature vector in $C P^{m}$ ) and in the case of submanifolds of low dimension or codimension, without any a priori assumptions at all. We also give some properties of 1-type submanifolds of $C P^{m}$ in general, and produce an extrinsic bound on the first eigenvalue of the Laplacian.

\section{$\S 1$. Preliminaries.}

Let $S^{2 m+1}=\left\{z \in C^{m+1} \mid \bar{z}^{t} z=1\right\}$ be the unit hypersphere centered at the origin. Then by identifying $z$ with $\lambda z$, where $\lambda \in C,|\lambda|=1$, we get the quotient space which defines $\boldsymbol{C} P^{m}$. We actually obtain the fiber bundle $\pi: S^{2 m+1} \rightarrow \boldsymbol{C} P^{m}$ with structure group $S^{1}$ whose vertical space at $v \in S^{2 m+1}$ is $\boldsymbol{R}\{i v\}$. Let $\mathcal{H}_{v}$ be the orthogonal complement of the vertical space so that $T_{v} S^{2 m+1}=\boldsymbol{R}\{i v\} \oplus \mathscr{H}_{v}$. One defines the metric on $C P^{m}$ such that $d \pi_{v}: \mathscr{K}_{v} \rightarrow T_{\pi(v)} C P^{m}$ is an isometry for every $v$. The complex structure $J$ of $C P^{m}$ is defined by means of the complex structure of $C^{m+1}$ and the uniqueness of the horizontal lift. Then $\pi$ is a Riemannian submersion with totally geodesic fibers and $C P^{m}$ is Kähler manifold with constant holomorphic sectional curvature 4.

Let $H(m+1)=\left\{P \in G L(m+1 ; C) \mid \bar{P}=P^{t}\right\}$ be the set of $(m+1) \times(m+1)$ Hermitian matrices. Equipped with the metric

$$
\langle P, Q\rangle=(1 / 2) \operatorname{tr}(P Q), \quad P, Q \in H(m+1)
$$

it becomes the usual Euclidean space $E^{N}$ of dimension $N=(m+1)^{2}$. It is well known that the map $\tilde{\phi}: S^{2 m+1} \rightarrow H(m+1)$ given by $\tilde{\phi}(z)=z \bar{z}^{t}$, where $z \in S^{2 m+1}$ is regarded as a column matrix, induces an isometric immersion $\phi: C P^{m} \rightarrow H(m+1)$ such that

$$
\phi\left(C P^{m}\right)=\left\{P \in H(m+1) \mid P^{2}=P, \operatorname{tr} P=1\right\} .
$$

$\phi\left(\boldsymbol{C} P^{m}\right)$ actually lies in the intersection of the hyperplane $H_{1}=\{P \in H(m+1) \mid \operatorname{tr} P=1\}$ and the hypersphere of $E^{N}$ with radius $r=\sqrt{m / 2(m+1)}$ centered at $I /(m+1), I$ being the identity matrix of $H(m+1)$. For more details on the embedding $\phi$ see [3], [10], [11], [13], [14].

By identifying $\boldsymbol{C} P^{m}$ with $\phi\left(C P^{m}\right)$, which we will do henceforth, the tangent and the normal space of $C P^{m}$ at a point $P \in C P^{m}$ are given respectively by

$$
\begin{aligned}
& T_{\mathscr{P}} \boldsymbol{C} P^{m}=\{X \in H(m+1) \mid X P+P X=X\} \\
& T_{\mathfrak{L}} \boldsymbol{C} P^{m}=\{Z \in H(m+1) \mid Z P=P Z\} .
\end{aligned}
$$

The second fundamental form of the embedding $\phi$ at a point $P$ is given by 


$$
\sigma(X, Y)=(X Y+Y X)(I-2 P)
$$

and it is known that $\sigma$ is parallel, $\bar{\nabla} \sigma=0$. We have also

$$
\langle\sigma(X, Y), I\rangle=0, \quad\langle\sigma(X, Y), P\rangle=-\langle X, Y\rangle .
$$

The complex structure $J$ and the shape operator $\bar{A}$ of $\phi$ at $P$ are given respectively by

$$
J X=\sqrt{-1}(I-2 P) X, \quad \bar{A}_{Z} X=(X Z-Z X)(I-2 P) .
$$

The following formula for the Weingarten map of $\phi$ in the direction of $\sigma(X, Y)$ is due to $\mathrm{A}$. Ros [11]

$$
\text { (2) } \bar{A}_{\sigma(X, Y)} V=2\langle X, Y\rangle V+\langle Y, V\rangle X+\langle X, V\rangle Y+\langle J Y, V\rangle J X+\langle J X, V\rangle J Y \text {. }
$$

Let $f: M^{n} \rightarrow E^{N}$ be an isometric immersion of a compact Riemannian manifold into a Euclidean space, and let $\Delta$ be the Laplace operator acting on smooth functions on $M$. An immersion $f$ (or a submanifold $M$ ) is said to be of 1-type if it can be decomposed as $f=f_{0}+f_{t}$, where $f_{0}=$ const and $\Delta f_{t}=\lambda_{t} f_{t}$. In particular, if $x: M^{n} \rightarrow C P^{m}$ is an isometric immersion of a compact manifold and $\tilde{x}=\phi \circ x: M^{n} \rightarrow H^{\prime}(m+1)$, we will study submanifolds of $C P^{m}$ with 1-type $\tilde{x}$, i.e. those for which $\tilde{x}=\tilde{x}_{0}+\tilde{x}_{t}$ where $\tilde{x}_{0}$ is a constant matrix in $H(m+1)$ and $\tilde{x}_{t}$ a nonconstant eigenfunction of the Laplacian (more precisely, each entry of the matrix $\tilde{x}_{t}$ is either zero or an eigenfunction from the same eigenspace).

For the immersions $M^{n} \stackrel{x}{\rightarrow} \boldsymbol{C} P^{m} \stackrel{\leftrightarrow}{\rightarrow} H(m+1)$ let $\tilde{\nabla}$ denote the Euclidean connection in $H(m+1) ; \bar{\nabla}, \bar{A}, \bar{D}$ denote the (Levi-Civita) connection on $C P^{m}$, Weingarten map and the connection in the normal bundle of $\phi ; \nabla, A, D$ denote the induced connection on $M$, Weingarten map and the connection in the normal bundle of the immersion $x$ and $h, \tilde{h}, H, \tilde{H}$ denote the second fundamental forms and the mean curvature vectors of the immersions $x$ and $\tilde{x}=\phi \circ x$ respectively. $\Gamma(T M)$ and $\Gamma\left(T^{\perp} M\right)$ will denote the set of all smooth sections (vector fields) of the tangent and normal bundle of $M$ respectively. We consider a local frame of orthonormal vector fields $e_{1}, \cdots, e_{n}, e_{n+1}, \cdots, e_{2 m}$ tangent to $C P^{m}$ where the first $n$ vectors are tangent to $M$ and the remaining ones are normal to $M$. In general, index $i$ will range from 1 to $n$ and index $r$ from $n+1$ to $2 m$, so that $e_{\imath}, e_{r}$ represent basis vectors tangent to $M$ and normal to $M$ respectively. All immersions are assumed smooth and all manifolds will be assumed compact, connected smooth Riemannian manifolds of dimension $\geqq 2$.

A submanifold $M$ of the complex projective space is called a $C R$-submanifold if the tangent bundle $T M$ splits into an orthogonal direct sum of two (differentiable) distributions $T M=\mathscr{D} \oplus \mathscr{D}^{\perp}$ such that $J \mathscr{D} \subset \mathscr{D}$ and $J \mathscr{D}^{\perp} \subset T^{\perp} M$. If $\mathscr{D}^{\perp}=\{0\}$ we have a complex (invariant) submanifold and if $\mathscr{D}=\{0\}$ we have a totally real one. 


\section{$\S 2$. 1-Type submanifolds of $C P^{m}$}

Given a submanifold $x: M^{n} \rightarrow \boldsymbol{C} P^{m}$. Then $\tilde{x}=\phi \circ x$ is a 1-type immersion if $\tilde{x}=\tilde{x}_{0}+\tilde{x}_{t}$ as above so that $\Delta \tilde{x}=\lambda_{t}\left(\tilde{x}-\tilde{x}_{0}\right)$. On the other hand,

$$
\Delta \tilde{x}=-n \tilde{H}=-n H-\sum_{i} \sigma\left(e_{i}, e_{i}\right)
$$

and therefore $\tilde{x}$ is of 1-type if and only if

$$
-n H-\sum_{i} \sigma\left(e_{i}, e_{\imath}\right)=\lambda_{t}\left(\tilde{x}-\tilde{x}_{0}\right) .
$$

Differentiating this formula along an arbitrary vector field $X \in \Gamma(T M)$, taking $\tilde{\nabla}_{X}$ of both sides we obtain (we assume for simplicity $\nabla_{X} e_{2}=0$ at a point where the calculation is done)

$$
n A_{H} X-n D_{X} H-n \sigma(X, H)+\sum_{\imath} \bar{A}_{\sigma\left(e_{i}, e_{i}\right)} X-\sum_{\imath} \bar{D}_{X} \sigma\left(e_{\imath}, e_{\imath}\right)=\lambda_{t} X .
$$

Because $\sigma$ is parallel we have

$$
\begin{aligned}
& \sum_{\imath} \bar{D}_{X} \sigma\left(e_{\imath}, e_{i}\right)=2 \sum_{\imath} \sigma\left(\bar{\nabla}_{X} e_{i}, e_{\imath}\right)=2 \sum_{\imath} \sigma\left(h\left(X, e_{\imath}\right), e_{\imath}\right)=2 \sum_{r} \sigma\left(A_{r} X, e_{r}\right), \\
& n \sigma(X, H)=\sum_{r}\left(\operatorname{tr} A_{r}\right) \sigma\left(X, e_{r}\right) .
\end{aligned}
$$

From (2) we also compute

$$
\begin{aligned}
\left.\sum_{\imath} \bar{A}_{\sigma\left(e_{i}, e_{i}\right.}\right) X & =\sum_{\imath}\left[2 X+2\left\langle e_{i}, X\right\rangle e_{i}-2\left\langle e_{\imath}, J X\right\rangle J e_{\imath}\right] \\
& =2(n+1) X-2 J(J X)_{T}
\end{aligned}
$$

where for a vector $W$ tangent to $C P^{m}, W_{T}$ and $W_{N}$ denote its orthogonal projections to the tangent and normal spaces $T M, T^{\perp} M$ at a given point respectively. Therefore, by comparing parts tangent and normal to $C P^{m}$ in (4) and using (5), (6) and (7) we obtain

LEMMA 1. Let $x: M^{n} \rightarrow \boldsymbol{C} P^{m}$ be an isometric immersion of a compact manifold. Then $\tilde{x}=\phi \circ x$ is of 1-type if and only if for every vector field $X \in \Gamma(T M)$

(8) $\quad n A_{H} X-n D_{X} H+2(n+1) X-2 J(J X)_{T}=\lambda_{t} X$ for some constant $\lambda_{t}$ and

(9) $\sum_{r} \sigma\left(B_{r} X, e_{r}\right)=0$, where $B_{r}=\left(\operatorname{tr} A_{r}\right) I+2 A_{r}$.

Because $\bar{A}_{z}=0 \Leftrightarrow Z=\rho I$ we obtain

LEMMA 2. With the assumptions as above, $\tilde{x}$ is of 1-type if and only if for any vector fields $X, Y \in \Gamma(T M)$ and $\xi \in \Gamma\left(T^{\perp} M\right)$ we have

$$
n A_{H} X-n D_{X} H+2(n+1) X-2 J(J X)_{T}=\lambda_{t} X, \lambda_{t}=\text { const }
$$




$$
\begin{aligned}
& \sum_{r}\left[\left\langle B_{r} X, Y\right\rangle e_{r}+\left\langle J e_{r}, Y\right\rangle J B_{r} X+\left\langle J B_{r} X, Y\right\rangle J e_{r}\right]=0, \\
& \sum_{r}\left[\left\langle e_{r}, \xi\right\rangle B_{r} X+\left\langle J e_{r}, \xi\right\rangle J B_{r} X+\left\langle J B_{r} X, \xi\right\rangle J e_{r}\right]=0 .
\end{aligned}
$$

Proof. First we claim that $\bar{A}_{Z}=0 \Leftrightarrow Z=\rho I$, where $\rho \in \boldsymbol{R}$ and $I$ is the $(m+1)$ $\times(m+1)$ identity matrix. If $Z=\rho I$ then obviously $\bar{A}_{z}=0$. Conversely, let us suppose that $\bar{A}_{Z} X=(X Z-Z X)(I-2 P)=0$ for every $X \in T_{P} C P^{m}$. After multiplying this formula by $I-2 P$ on the right we see that $X Z=Z X$. Because the immersion is equivariant it is enough to prove the claim at the "origin"

$$
P_{0}=\left(\begin{array}{llll}
1 & & & \\
& 0 & & \\
& & \ddots & \\
& & & 0
\end{array}\right) .
$$

Any vector $X$ tangent to $C P^{m}$ at $P_{0}$ can be represented as $X=\left(\begin{array}{ll}0 & \bar{b}^{t} \\ b & \bar{b}\end{array}\right)$, where $b \in \boldsymbol{C}^{m}$, and $\mathbf{0}$ is the $m \times m$ zero matrix, and a vector $Z$ normal to $\boldsymbol{C} P^{m}$ at $P_{0}$ has the form

$$
Z=\left(\begin{array}{ll}
\rho & 0 \\
0 & S
\end{array}\right) \text {, where } \rho \in \boldsymbol{R} \text { and } S \in H(m) \quad \text { (see }[10] \text { ). }
$$

Condition $X Z=Z X$ for every $X \in T C P^{m}$ implies then that $S b=\rho b$ for every $b \in C^{m}$, hence $S=\rho I$ and in turn $Z=\rho I$. Now we show that (9) is equivalent to (10) and (11) together. First observe that by (2), formulas (10) and (11) are equivalent to $\sum_{r} \bar{A}_{\sigma\left(B_{r} X, e_{r}\right)} Y=0$ and $\sum_{r} \bar{A}_{\sigma\left(B_{r} X, e_{r}\right)} \xi=0$ respectively. However, if $\sum_{r} \bar{A}_{\sigma\left(B_{r} X, e_{r}\right)}=0$ then from the above we have $\sum_{r} \sigma\left(B_{r} X, e_{r}\right)=\rho I$, but since $\langle\sigma(V, W), I\rangle=0$ we obtain (9). If (9) is true then by tracing this argument backwards we obtain (10) and (11).

It is easy to see now that if $\tilde{x}$ is of 1-type, the conditions that $M$ has parallel mean curvature vector in $C P^{m}$ and that it is a $C R$ submanifold of $C P^{m}$ are equivalent. Namely, if $M$ is a $C R$ submanifold then $J(J X)_{T}$ is always tangent to $M$ and therefore from (8) $D H=0$. Conversely, suppose $D H=0$ (so that the mean curvature $\alpha$ is constant), and let $X$ be a principal direction of $A_{H}$ i.e. $A_{H} X=\mu X$. Then from (8) we get $2(J X)_{T}=\left[\lambda_{t}-2(n+1)-\mu n\right] J X$, so that at any given point we have either

$1^{\circ}$ $\mu=\frac{1}{n}\left[\lambda_{t}-2(n+1)\right]$, equivalently $(J X)_{T}=0$ or

$2^{\circ}$.

$$
\mu=\frac{1}{n}\left[\lambda_{t}-2(n+2)\right], \text { equivalently }(J X)_{N}=0
$$


Therefore, there are at most two distinct principal curvatures $\mu_{1}=\frac{1}{n}\left[\lambda_{t}-2(n+2)\right]$ with multiplicity $p$ and $\mu_{2}=\frac{1}{n}\left[\lambda_{t}-2(n+1)\right]$ with multiplicity $q$. Moreover,

$$
n \alpha^{2}=\operatorname{tr} A_{H}=\frac{p}{n}\left[\lambda_{t}-2(n+2)\right]+\frac{q}{n}\left[\lambda_{t}-2(n+1)\right]=\lambda_{t}-2(n+2)+\frac{2 q}{n},
$$

and hence $p$ and $q$ are constant. Let

$$
\begin{aligned}
& \mathscr{D}=\left\{X \in T M \mid A_{H} X=\mu_{1} X\right\}=\left\{X \in T M \mid(J X)_{N}=0\right\} \text { and } \\
& \mathscr{D}^{\perp}=\left\{X \in T M \mid A_{H} X=\mu_{2} X\right\}=\left\{X \in T M \mid(J X)_{T}=0\right\} .
\end{aligned}
$$

Then $J \mathscr{D}=\mathscr{D}$ and $J \mathscr{D}^{\perp} \subset T^{\perp} M$ and consequently $M$ is a $C R$ submanifold. We are now ready to prove the main theorem

THEOREM 1. Let $x: M^{n} \rightarrow \boldsymbol{C} P^{m}$ be an isometric immersion of a compact Riemannian manifold into $\boldsymbol{C P}^{m}$ with parallel mean curvature vector (or let $M$ be a CR submanifold of $\left.C P^{m}\right)$. Then $\tilde{x}=\phi \circ x$ is of 1-type if and only if one of the following cases occurs

1) $n$ is even, and $M^{n}$ is congruent to the complex projective space $C P^{n / 2}$ immersed as a totally geodesic complex submanifold of $\boldsymbol{C} P^{m}$.

2) $M^{n}$ is a totally real minimal submanifold of a complex totally geodesic $\boldsymbol{C} P^{n}$ in $\boldsymbol{C} P^{m}$.

3) $n$ is odd, and $M^{n}$ is congruent to the geodesic hypersphere

$$
M_{0,(n-1) / 2}^{C_{1}}=\pi\left(S^{1}(\sqrt{1 /(n+3)}) \times S^{n}(\sqrt{(n+2) /(n+3)})\right)
$$

of the complex projective space $\boldsymbol{C} P^{(n+1) / 2}$ immersed as a totally geodesic complex submanifold of $\boldsymbol{C P}^{m}$.

Proof. Any of the cases 1), 2) and 3) is indeed of 1-type as follows from Theorem A of Ros and the result of [8] (also from our Lemma 2). Now assume that the submanifold of $C P^{m}$ with $D H=0$ is of 1-type. As shown above such submanifold is also $C R$, so that at each point of $M$ we have the following orthogonal splitting of the tangent space of $C P^{m}$.

$$
T C P^{m}=\mathscr{D} \oplus \mathscr{D}^{\perp} \oplus J \mathscr{D}^{\perp} \oplus \nu
$$

where $\mathscr{D}$ and $\nu$ are holomorphic subbundles and $T M=\mathscr{D} \oplus \mathscr{D}^{\perp}, T^{\perp} M=J \mathscr{D}^{\perp} \oplus \nu$. Let $p=2 k=\operatorname{dim} \mathscr{D}$ and $q=\operatorname{dim} \mathscr{D}^{\perp}$ so that $p+q=n$ and let $e_{1}, \cdots, e_{p}, e_{p+1}, \cdots$, $e_{p+q}, e_{n+1}, \cdots, e_{n+q}, e_{n+2+1}, \cdots, e_{2 m}$ be a local orthonormal basis for $T C P^{m}$ adapted to the decomposition (12) where $J e_{p+j}=e_{n+\jmath}$ for $j=1, \cdots, q$. Because $\nu$ is a holomorphic subbundle we can assume that $\left\{e_{n+q+1}, \cdots, e_{2 m}\right\}$ is a $J$-basis for $\nu$ i.e. it is equal to $\left\{e_{s}, e_{s *}=J e_{s}\right\}, s=n+q+1, \cdots, m+k+q$. Now in formula (11) let $\xi=e_{s}$, where $s$ is any index in the range $n+q+1, \cdots, m+k+q$. Then (11) reduces to 


$$
0=\sum_{r}\left[\left\langle e_{r}, e_{s}\right\rangle B_{r} X+\left\langle J e_{r}, e_{s}\right\rangle J B_{r} X\right]=B_{s} X-J B_{s *} X
$$

Hence, $B_{s} X=J B_{s *} X$ and $B_{s *} X=-J B_{s} X$ for a $J$-basis vector $e_{s}$ in $\nu$. We now show that $A_{s}=A_{s *}=0$. Using the above we compute the trace of $B_{s}$ as

$$
(n+2) \operatorname{tr} A_{s}=\operatorname{tr} B_{s}=\sum_{\imath}\left\langle B_{s} e_{\imath}, e_{\imath}\right\rangle=\sum_{\imath}\left\langle J B_{s *} e_{\imath}, e_{\imath}\right\rangle=0
$$

because $B_{s *}$ is symmetric and $J$ skew symmetric. So $\operatorname{tr} A_{s}=0$ and similarly $\operatorname{tr} A_{s *}=0$. Note that at this point we can conclude that $H \in J \mathscr{D}^{\perp}$ since $\operatorname{tr} A_{r}=0$ for any $e_{r} \in \nu$. Moreover, from the above we get

$$
A_{s *} X=-J A_{s} X \text {. }
$$

Let $Y \in \mathscr{D}^{\perp}$ be arbitrary. The formula (10) becomes

$$
\sum_{r}\left[\left\langle B_{r} X, Y\right\rangle e_{r}+\left\langle J e_{r}, Y\right\rangle J B_{r} X\right]=0 .
$$

Fix $e_{s} \in \nu$ and take the inner product of the above formula with $e_{s}$ to get $\left\langle B_{s} X, Y\right\rangle=0$ for every $Y \in \mathscr{D}^{\perp}$. This implies $B_{s} X \in \mathscr{D}$ and hence $A_{s} X \in \mathscr{D}$ for any $X$ tangent to $M$ and any $e_{s} \in \nu$, so that $J A_{s} X \in \mathscr{D}$ as well.

On the other hand, because $C P^{m}$ is Kähler we have for $e_{s} \in \nu$ (note also $\left.e_{s *} \in \nu\right) \bar{\nabla}_{X}\left(J e_{s}\right)=J \bar{\nabla}_{X} e_{s}$, i.e.

$$
-A_{s *} X+D_{X} e_{s *}=-J A_{s} X+J D_{X} e_{s},
$$

where both $A_{s *} X$ and $J A_{s} X$ are tangent to $M$ and $D_{X} e_{s^{*}}$ is normal to $M$. Again choosing $Y \in \mathscr{D}^{\perp}$ we obtain from (15)

$$
\left\langle J D_{X} e_{s}, Y\right\rangle=\left\langle J D_{X} e_{s}-J A_{s} X, Y\right\rangle=\left\langle D_{X} e_{s *}-A_{s *} X, Y\right\rangle=0 .
$$

Obviously $\left\langle J D_{X} e_{s}, Y\right\rangle=0$ for every $Y \in \mathscr{D}$ so that $J D_{X} e_{s}$ is normal to $M$ (actually it belongs to $\nu$ ). Comparing parts tangent to $M$ in (15) we conclude $A_{s *} X=J A_{s} X$. But together with (13) this implies $A_{s^{*}}=A_{s}=0$.

We can assume that the constant mean curvature $\alpha$ is not zero because if it is, we have a $C R$-minimal submanifold of 1-type and therefore by Theorem A cases 1 ) or 2) occur. Since $H \in J D^{\perp}$ we also assume from now on that the unit normal vector $e_{n+1}$ is in the direction of $H$ so that in the basis of principal directions the Weingarten map $A_{n+1}$ has the following block form

$$
A_{n+1}=\left(\begin{array}{cc}
\beta I_{p} & 0 \\
0 & \gamma I_{q}
\end{array}\right) \text {, where } p=\operatorname{dim} \mathscr{D}, q=\operatorname{dim} \mathscr{D}^{\perp}
$$

and $\beta=(1 / n \alpha)\left[\lambda_{t}-2(n+2)\right], \gamma=(1 / n \alpha)\left[\lambda_{t}-2(n+1)\right]$. From $\operatorname{tr} A_{n+1}=n \alpha$ we get $\beta=\alpha-2 q / n^{2} \alpha$ and $\gamma=\alpha+2 p / n^{2} \alpha$. Next we show that $q=\operatorname{dim} \mathscr{D}^{\perp}=1$. Let $Y \in \mathscr{D}$ in formula (10) so that

$$
\sum_{r}\left[\left\langle B_{r} X, Y\right\rangle e_{r}+\left\langle J B_{r} X, Y\right\rangle J e_{r}\right]=0 .
$$


Because $B_{r}=0$ for $r \geqq n+q+1$ and $J e_{r} \in \mathscr{D}^{\perp}$ for $n+1 \leqq r \leqq n+q$ we obtain $\left\langle B_{r} X, Y\right\rangle=0$ i.e. $B_{r} X \in \mathscr{D}^{\perp}$ for every $X \in \Gamma(T M)$ and every $r$. In particular, for $X \in \mathscr{D}$ we get (if $\mathscr{D}=\{0\}$ there is no $\beta$ ) $B_{n+1} X=n \alpha X+2 A_{n+1} X=(n \alpha+2 \beta) X \in \mathscr{D}^{\perp}$ so that $n \alpha+2 \beta=0$ i.e.

$$
\beta=\alpha-2 q / n^{2} \alpha=-n \alpha / 2 \text {. }
$$

For $r=n+j, \jmath=2, \cdots, q$ we obtain $B_{n+\jmath} X=2 A_{n+\jmath} X \in \mathscr{D}^{\perp}$ for every $X \in \Gamma(T M)$ and hence $0=\left\langle A_{n+j} \mathscr{D}^{\perp}, \mathscr{D}\right\rangle=\left\langle\mathscr{D}^{\perp}, A_{n+j} \mathscr{D}\right\rangle$ so that $A_{n+j} \mathscr{D}=0$, which implies that $A_{n+\jmath}$ has the block form

$$
A_{n+\jmath}=\left(\begin{array}{cc}
0 & 0 \\
0 & M_{j}
\end{array}\right)
$$

where $M_{\jmath}$ is $q \times q$ symmetric matrix with $\operatorname{tr} M_{\jmath}=\operatorname{tr} A_{n+\jmath}=0$. Furthermore, it is known from [2] that

$$
A_{J X} Y=A_{J Y} X \quad \text { for } \quad X, Y \in \mathscr{D}^{\perp} \text {. }
$$

Letting $X=e_{p+1}, Y=e_{p+\jmath}, 2 \leqq j \leqq q$ in this formula we get $A_{n+j} e_{p+1}=A_{n+1} e_{p+\jmath}=$ $\gamma e_{p+j}$ and therefore $M$, has the form

$$
M_{\jmath}=\left(\begin{array}{ccccc}
0 & \cdots & \gamma & \cdots & 0 \\
\vdots & & & & \\
\gamma & & * & & \\
\vdots & & & & \\
0 & & & &
\end{array}\right)
$$

where $\gamma$ is in the $j^{\text {th }}$ slot in the first row and the first column and all other entries there are 0 . We now show that $q=\operatorname{dim} \mathscr{D}^{\perp}=1$. Suppose that $q \geqq 2$. Then for $j \geqq 2$ we have $B_{n+\jmath}=2 A_{n+\rho}$ and from the above $\left\langle B_{n+j} e_{p+\jmath}, e_{p+1}\right\rangle=$ $\left\langle e_{p+\jmath}, B_{n+j} e_{p+1}\right\rangle=2 \gamma$. On the other hand, putting $X=e_{p+\jmath}, Y=e_{p+1}$ in (10) we have

$$
0=\sum_{r}\left\langle B_{r} e_{p+\jmath}, e_{p+1}\right\rangle e_{r}-J B_{n+1} e_{p+\jmath}=\sum_{r}\left\langle B_{r} e_{p+\jmath}, e_{p+1}\right\rangle e_{r}-(n \alpha+2 \gamma) e_{n+\jmath}
$$

and by comparing $e_{n+j}$-components we obtain $\left\langle B_{n+j} e_{p+j}, e_{p+1}\right\rangle=n \alpha+2 \gamma$ so that $\alpha=0$. Since we assumed nonzero mean curvature this contradiction proves $q=1$ i. e. purely real distribution is one-dimensional and $n=2 k+1$ is odd. Since $\operatorname{dim} M \geqq 2$ it follows that $\operatorname{dim} \mathscr{D} \neq 0$. Then from (16) we find $\alpha=2 / n \sqrt{n+2}$ and

$$
A_{n+1}=\frac{1}{\sqrt{n+2}}\left(\begin{array}{cc}
-I_{n-1} & 0 \\
0 & n+1
\end{array}\right), A_{r}=0 \quad \text { for } r \geqq n+2 .
$$

Therefore, the first normal space equals $N_{1}=\operatorname{Im} h=J \mathscr{D}^{\perp}=\boldsymbol{R}\{H\}$ and since $D H=0, N_{1}$ is parallel, i.e. $D N_{1} \subset N_{1}$. Also, $J\left(T M \oplus N_{1}\right)=T M \oplus N_{1}$ and therefore by the reduction theorem of $\mathrm{B}$. Opozda [12, Prop. 3.1] there exists a totally 
geodesic complex submanifold $\bar{M}$ of $C P^{m}$ so that the image of $M$ is contained in $\bar{M}$ and $T_{p} \bar{M}=T_{p} M \oplus N_{1 p}$ for every $p \in M$. Note that even though Opozda's theorem is formaly proved for totally real submanifolds, it is clear that her proof holds for $C R$ submanifolds verbatim. The idea is as follows. Choose $p \in M$ and $v \in \pi^{-1}(p)$ and define $K_{v}=\left(\pi_{*} \mid \mathscr{A}_{v}\right)^{-1}\left(T_{p} M \oplus N_{1 p}\right) \oplus \operatorname{Span}_{C}\{v\}$. Then $K_{v}$ is a complex subspace of $\boldsymbol{C}^{m+1}$. Set $\bar{M}_{v}=\pi\left(K_{v} \cap S^{2 m+1}\right)$ so that $\bar{M}_{v}$ is a totally geodesic complex submanifold of $C P^{m}$ and $T_{p} \bar{M}=T_{p} M \oplus N_{1 p}$. From the conditions above it follows then that $\bar{M}_{v}$ does not depend on $p$ and $v \in \pi^{-1}(p)$; see also [1]. This means that $M$ is a (real) hypersurface of some complex totally geodesic $C P^{(n+1) / 2}$ and by [8] $M$ is the geodesic hypersphere of the case 3) which completes the proof.

At the present time we are not able to give the classification of 1-type submanifolds of $C P^{m}$ without any a priori assumptions but some general properties of those are given in the next theorem.

THEOREM 2. If $M$ is a 1-type submanifold of $\boldsymbol{C P}^{m}$ with the mean curvature vector $H$, then the mean curvature $\alpha$ of the immersion is constant, JH is tangent to $M$ and integral curves of $J H$ are geodesics (if $H \neq 0$ ).

Proof. As before, since $B_{r}$ is symmetric and $J$ skew-symmetric we have $\sum_{\imath}\left\langle J B_{r} e_{\imath}, e_{\imath}\right\rangle=0$ and putting $X=Y=e_{\imath}$ in (10) and adding on $i=1, \cdots, n$ we obtain

and therefore

$$
\begin{aligned}
0 & =\sum_{r}\left(\operatorname{tr} B_{r}\right) e_{r}+\sum_{r, \imath}\left\langle J e_{r}, e_{\imath}\right\rangle J B_{r} e_{\imath} \\
& =n(n+2) H+\sum_{r} J B_{r}\left(J e_{r}\right)_{T},
\end{aligned}
$$

$$
J H=\frac{1}{n(n+2)} \sum_{r} B_{r}\left(J e_{r}\right)_{T} \in T M .
$$

Now taking the inner product of (8) with $H$ we get

$$
n\left\langle D_{X} H, H^{\prime}\right\rangle=-2\left\langle J(J X)_{T}, H\right\rangle=2\left\langle(J X)_{T}, J H\right\rangle=2\langle J X, J H\rangle=2\langle X, H\rangle=0,
$$

hence $X\langle H, H\rangle=X \alpha^{2}=0$ for every tangent vector $X$, and therefore the mean curvature $\alpha$ is constant. Letting $X=J H$ in (8) we obtain

$$
D_{J H} H=0, \quad A_{H} J H=\frac{1}{n}\left[\lambda_{t}-2(n+1)\right] J H .
$$

Therefore,

$$
\bar{\nabla}_{J H}(J H)=J \bar{\nabla}_{J H} H=-J A_{H} J H+J D_{J H} H=\frac{1}{n}\left[\lambda_{t}-2(n+1)\right] H .
$$

On the other hand $\bar{\nabla}_{J H}(J H)=\nabla_{J H}(J H)+h(J H, J H)$, and we conclude $\nabla_{J H}(J H)=0$ i. e. the integral curves of $J H$ are geodesics. 
By way of a remark we note that having proved that $\alpha=$ const for 1 -type submanifolds of $C P^{m}$, in Th. 1 instead of $D H=0$ we could have made slightly weaker assumption (if $\alpha \neq 0$ ) that the normalized mean curvature vector is parallel i.e. $D e_{n+1}=0$.

CoRollary 1. Let $x: M^{2} \rightarrow C P^{m}$ be an isometric immersion of a closed surface in $\boldsymbol{C} P^{m}$. Then $\tilde{x}=\phi \circ x$ is of 1-type if and only if one of the cases 1) or 2) of Theorem 1 occurs. The same conclusion holds if $M$ is a 1-type submanifold of codimension two.

Proof. We assume $H \neq 0$, otherwise $M$ is minimal and we proceed as before to show that $M$ is one of the cases 1) or 2). By Theorem 2, JH is tangent to $M$ and is a principal direction of $A_{H}$. Let $U \perp J H$ be the other principal direction of $A_{H}$ i.e. $A_{H} U=\mu U$. Then $U$ and $J H$ span $T M$, and $\langle J U, U\rangle=0$, $\langle J U, J H\rangle=\langle U, H\rangle=0$ and consequently $J U \in T^{\perp} M$. Since also $J J H \in T^{\perp} M$ we conclude that $M$ is a totally real surface and hence of case 2) of Theorem 1.

If $M$ is codimension two submanifold, let $H, \xi$ span $T^{\perp} M(\xi \perp H)$. Then since $J H^{\prime}$ is tangent to $M$ we have $\langle J \xi, H\rangle=-\left\langle\xi, J H^{\prime}\right\rangle=0$ and $\langle J \xi, \xi\rangle=0$ so that $J \xi$ is also tangent to $M$. This implies that $M$ is a $C R$-submanifold and therefore by Theorem 1 one of the cases 1 ) and 2).

We now want to carry out investigation of 1-type submanifolds of $\boldsymbol{C} P^{m}$ without any a priori assumptions. As before, we can show that there are at most two distinct principal curvatures of $A_{H}$ and corresponding two distributions $\mathscr{D}=\operatorname{Ker} B_{n+1}$ and $\mathscr{D}^{\perp}=\operatorname{Im} B_{n+1}$ with $J \mathscr{D}^{\perp} \subset T^{\perp} M$, but we are not able to show $J \mathscr{D \subset} T M$.

Let $Y=J e_{n+1}$ in (10) which then becomes $\sum_{r}\left\langle B_{r} X, J e_{n+1}\right\rangle e_{r}+J B_{n+1} X=0$ from where we obtain

$$
J B_{n+1} X=-\sum_{r}\left\langle B_{r} X, J e_{n+1}\right\rangle e_{r} \in T^{\perp} M .
$$

If we define $\mathscr{D}^{\perp}=\left\{X \in T M \mid J X \in T^{\perp} M\right\}$ then we see that $\operatorname{Im} B_{n+1} \subset \mathscr{D}^{\perp}$. At each point of $M$ we have the orthogonal decomposition

$$
T M=\operatorname{Ker} B_{n+1} \oplus \operatorname{Im} B_{n+1} .
$$

Let $e_{1}, \cdots, e_{p}$ be an orthonormal basis for $\operatorname{Ker} B_{n+1}$; these are eigenvectors for eigenvalue 0 . The remaining orthonormal eigenvectors $e_{p+1}, \cdots, e_{p+q}$ of $B_{n+1}$ span $\operatorname{Im} B_{n+1}$. Because $B_{n+1}=(n \alpha) I+2 A_{n+1}$ we see that $e_{1}, \cdots, e_{p}$ are eigenvectors of $A_{n+1}$ for eigenvalue $\beta=-n \alpha / 2$ and $e_{p+1}, \cdots, e_{n}$ are also eigenvectors of $A_{n+1}$ corresponding to eigenvalue $\gamma=(1 / n \alpha)\left[\lambda_{t}-2(n+1)\right]$ (This follows from (8) and (19)). In other words

$$
A_{n+1}=\left(\begin{array}{cc}
\beta I_{p} & 0 \\
0 & \gamma I_{q}
\end{array}\right) \text {, where } p=\operatorname{dim} \operatorname{Ker} B_{n+1}, q=\operatorname{dim} \operatorname{Im} B_{n+1} .
$$


Moreover, $p$ and $q$ are constant because $p+q=n$ and $p \beta+q \gamma=n \alpha=$ const $(\beta \neq \gamma$, see below). If $\alpha=0$ then the submanifold is $C R$ and therefore classified by Theorem A. From now on we assume $\alpha \neq 0$. Not all eigenvalues of $A_{n+1}$ can be equal to $\beta=-n \alpha / 2$ because if that is the case we would have $n \alpha=\operatorname{tr} A_{n+1}$ $=-n^{2} \alpha / 2$ which implies $\alpha=0$. Also not all eigenvalues can be equal to $\gamma=(1 / n \alpha)\left[\lambda_{t}-2(n+1)\right]$ because if it is so it would follow that $(J X)_{T}=0$ for every $X$ tangent to $M$ and hence $M$ would be totally real submanifold and therefore minimal by Theorem 1. It is also clear now that $\operatorname{Im} B_{n+1}=\mathscr{D}^{\perp}$ because if $\operatorname{Im} B_{n+1}$ is a proper subspace of $\mathscr{D}^{\perp}$ there would exist a vector $X \in \operatorname{Ker} B_{n+1}$ satisfying $J X \in T^{\perp} M$. Then (8) implies $A_{n+1} X=(1 / n \alpha)\left[\lambda_{t}-2(n+1)\right] X$. On the other hand for $X \in \operatorname{Ker} B_{n+1}$ we have $A_{n+1} X=-(n \alpha / 2) X$ which is a contradiction proving the claim. In particular we can set $e_{p+1}=-J e_{n+1} \in \operatorname{Im} B_{n+1}$. Let us also denote $\mathscr{D}=\operatorname{Ker} B_{n+1}$. With respect to the decomposition (20) $B_{n+1}$ has the following block form

$$
B_{n+1}=\left(\begin{array}{cc}
0 & 0 \\
0 & (n \alpha+2 \gamma) I_{q}
\end{array}\right) .
$$

We now prove the following lemma which enables us to classify 1-type submanifolds of $C P^{m}$ for which $q=\operatorname{dim} \operatorname{Im} B_{n+1}=1$.

LEMMA 3. Let $M^{n}$ be a 1-type submanifolds of $\boldsymbol{C} P^{m}$ for which $q=1$. Then $M^{n}$ has parallel mean curvature vector.

Proof. Let $X \in \mathscr{D}=\operatorname{Ker} B_{n+1}$ and $\xi=J e_{p+1}=e_{n+1}$ in (11). We get

$$
B_{n+1} X+\sum_{r}\left\langle B_{r} X, e_{p+1}\right\rangle J e_{r}=0
$$

and consequently $B_{r} X \perp e_{p+1}$. It follows immediately that also

$$
A_{r} X \perp e_{p+1}, \quad \text { for every } r=n+1, \cdots, 2 m \text { and every } X \in \mathscr{D} .
$$

Further, by putting $X=Y=e_{p+1}=-J e_{n+1}$ in (10) we get

$$
\begin{aligned}
0 & =\sum_{r}\left\langle B_{r} e_{p+1}, e_{p+1}\right\rangle e_{r}-J B_{n+1} e_{p+1} \\
& =\sum_{r}\left\langle B_{r} e_{p+1}, e_{p+1}\right\rangle e_{r}-(n \alpha+2 \gamma) e_{n+1} \\
& =\sum_{r \geq n+2}\left\langle B_{r} e_{p+1}, e_{p+1}\right\rangle e_{r}
\end{aligned}
$$

and therefore $B_{r} e_{p+1} \perp e_{p+1}$ for $r \geqq n+2$. Because $B_{r}=2 A_{r}$ we also have

$$
A_{r} e_{p+1} \perp e_{p+1} \quad \text { for every } \quad r=n+2, \cdots, 2 m
$$

We recall the Codazzi equation (see e.g. [3])

$$
\left(\bar{\nabla}_{X} h\right)(Y, Z)-\left(\bar{\nabla}_{Y} h\right)(X, Z)=(\bar{R}(X, Y) Z)_{N}, \quad X, Y, Z \in \Gamma(T M),
$$


where the curvature tensor $\bar{R}(X, Y) Z$ of $C P^{m}$ is given by.

(25) $\bar{R}(X, Y) Z=\langle Y, Z\rangle X-\langle X, Z\rangle Y+\langle J Y, Z\rangle J X-\langle J X, Z\rangle J Y+2\langle X, J Y\rangle J Z$.

Let $X=Z=e_{p+1}, Y=e_{i}, i=1, \cdots, p$. Then from (25) we get

$$
\bar{R}\left(e_{p+1}, e_{i}\right) e_{p+1}=-e_{i}+3\left\langle J e_{i}, e_{p+1}\right\rangle e_{n+1}=-e_{i}
$$

so that the Codazzi equation (24) yields

$$
\begin{aligned}
0= & \left(\bar{\nabla} e_{p+1} h\right)\left(e_{i}, e_{p+1}\right)-\left(\bar{\nabla} e_{i} h\right)\left(e_{p+1}, e_{p+1}\right) \\
= & D_{e_{p+1}}\left(h\left(e_{i}, e_{p+1}\right)\right)-h\left(\nabla e_{p+1} e_{i}, e_{p+1}\right)-h\left(e_{i}, \nabla e_{p+1} e_{p+1}\right) \\
& -D_{e_{i}}\left(h\left(e_{p+1}, e_{p+1}\right)\right)+2 h\left(\nabla e_{i} e_{p+1}, e_{p+1}\right) .
\end{aligned}
$$

We have $\nabla e_{p+1} e_{p+1}=0$ by Theorem $2, h\left(e_{i}, e_{p+1}\right)=\sum_{r}\left\langle A_{r} e_{i}, e_{p+1}\right\rangle e_{r}=0$ by (22), $h\left(e_{p+1}, e_{p+1}\right)=\gamma e_{n+1}$ by (21) and (23) and the equation above becomes

$$
0=-\omega_{i}^{k}\left(e_{p+1}\right) h\left(e_{k}, e_{p+1}\right)-D e_{i}\left(\gamma e_{n+1}\right)+2 \omega_{p+1}^{k}\left(e_{i}\right) h\left(e_{k}, e_{p+1}\right)
$$

where $\omega_{i}^{k}$ denote the connection 1 -forms and summation is on $k=1, \cdots, n$. Because $q=1, p=n-1, \beta=-n \alpha / 2$, we find $\gamma=\alpha n(n+1) / 2=$ const $\neq 0$. Now using (21), (22), (23) and (26) we have

$$
-\omega_{i}^{p+1}\left(e_{p+1}\right) \gamma e_{n+1}=\gamma D_{e_{i}} e_{n+1},
$$

and because $\gamma \neq 0$, and $e_{n+1} \perp D_{e_{i}} e_{n+1}$ we obtain $D_{e_{i}} e_{n+1}=0, i=1, \cdots, p$. In other words $D_{X} H=0$ for $X \in \mathscr{D}=\operatorname{Ker} B_{n+1}$ and from (8) we also have $D_{X} H=0$ for $X \in \mathscr{D}^{\perp}$ and therefore $D H=0$ and $M$ is a $C R$-submanifold.

COROLlARY 2. Let $x: M^{3} \rightarrow C P^{m}$ be a compact 3-dimensional submanifold. Then $\tilde{x}=\phi \circ x$ is of 1-type if and only if $M^{3}$ is one of the cases 2) or 3) of Theorem 1 (with $n=3$ ).

Proof. If $M$ is minimal the classification follows as before. If $\alpha \neq 0$ suppose first that $p=\operatorname{dim} \mathscr{D}=2, q=\operatorname{dim} \mathscr{D}^{\perp}=1$. Then Lemma 3 and Theorem 1 give desired classification. If $p=1$, and $q=2$, let $\mathscr{D}=\operatorname{Span}\{X\}, \mathscr{D}^{\perp}=\operatorname{Span}\{Y, Z\}$. In that case $\langle J X, X\rangle=0,\langle J X, Y\rangle=-\langle X, J Y\rangle=0$ and likewise $\langle J X, Z\rangle=0$, which implies that $M$ is a totally real submanifold and Theorem 1 proves the claim again.

The same idea can be used to classify codimension 3 submanifolds of $\boldsymbol{C} P^{m}$ which are of 1-type. Again, they are of three cases described in Theorem 1.

\section{§3. Remarks and related results}

1. The complete classification in Theorem 1 is not given because the classification of minimal totally real submanifolds $M^{n}$ of $C P^{n}$ is not known, and 
seemingly there are quite a few of them. For example, in [7] it was shown that the torus $T^{2}=S^{1} \times S^{1}$ can be minimally immersed in $C P^{2}$ as a totally real submanifold. Also, according to Naitoh $[9, \S 5]$ there are totally real minimal submanifolds $M^{n}$ of $C P^{n}$ including $S U(k) / S O(k)(n=(1 / 2)(k+2)(k-1)), S U(2 k) /$ $S p(k)\left(n=2 k^{2}-k-1\right), S U(k) \times S U(k) / S U(k)\left(n=k^{2}-1\right) E_{6} / F_{4}(n=26)$, and Ejiri [5] shows that certain circle bundles (tubes) over $S^{2}$ of sufficiently high even Chern number can be minimally immersed in $C P^{3}$ as a totally real submanifolds. Note however that there are some pinching theorems stating that under certain conditions on curvature (e.g. sectional curvature $K>0$ ) every totally real minimal submanifold $M^{n}$ of $C P^{n}$ is necessarily totally geodesic; see [16], [4], [6].

2. Study of finite type immersions often provides some information on the spectrum of the Laplacian on $M$. For example, if $M^{n}$ is a compact manifold that can be immersed into $C P^{m}$ with constant mean curvature $\alpha$ then the first nonzero eigenvalue of the Laplacian satisfies

$$
\lambda_{1} \leqq n \alpha^{2}+2(n+2)
$$

and the equality holds if and only if $M^{n}=C P^{n / 2}$ and the immersion is totally geodesic in $C P^{m}$ (in which case $\alpha=0$ ). Namely, if $\tilde{x}=\sum_{t=0}^{\infty} \tilde{x}_{t}, \Delta \tilde{x}_{t}=\lambda_{t} \tilde{x}_{t}, \quad \lambda_{0}=0$ then

$$
\int_{M}\langle\Delta \tilde{x}, \Delta \tilde{x}\rangle d V-\lambda_{1} \int_{M}\langle\Delta \tilde{x}, \tilde{x}\rangle d V=\sum_{t \geq 1} \lambda_{t}\left(\lambda_{t}-\lambda_{1}\right) \int_{M}\left\langle\tilde{x}_{t}, \tilde{x}_{t}\right\rangle d V \geqq 0,
$$

equality holding if and only if $\tilde{x}$ is of 1-type of order $\{1\}$. On the other hand, because $\Delta \tilde{x}=-n H-\sum_{i} \sigma\left(e_{i}, e_{2}\right)$, by using the equality (2.11) of [8] and (1) we get $\langle\Delta \tilde{x}, \tilde{x}\rangle=n$ and

$$
\langle\Delta \tilde{x}, \Delta \tilde{x}\rangle=n^{2} \alpha^{2}+2 n(n+1)+2 \sum_{\imath}\left\langle\left(J e_{i}\right)_{T},\left(J e_{\imath}\right)_{T}\right\rangle \leqq n^{2} \alpha^{2}+2 n(n+2),
$$

equality holding if and only if $(J X)_{N}=0$ for every $X \in \Gamma(T M)$, which implies that $M$ is an invariant submanifold, thus $C R$, so that Theorem 1 proves the claim (cf. [3, p. 314], [10]). Actually, it is easy to see from the above that for any compact submanifold $M^{n}$ of $C P^{m}(4)$ we have

$$
\lambda_{1} \leqq 2(n+2)+\frac{n}{\operatorname{vol}(M)} \int_{M} \alpha^{2} d V .
$$

3. Let $\phi: R P^{m} \rightarrow S M(m+1)$ be the first standard embedding of the real projective space into the set of symmetric matrices of degree $m+1$. There is a problem of determining all submanifolds $x: M^{n} \rightarrow \boldsymbol{R} P^{m}$ which are of 1-type via $\phi$, i.e. for which $\tilde{x}=\phi \circ x$ is a 1-type immersion. Chen [3] shows that the only complete 1-type submanifold of $\boldsymbol{R} P^{m}$ which is minimal in $\boldsymbol{R} P^{m}$ is the totally geodesic $\boldsymbol{R} P^{n}$. Because of the corresponding result for $C R$ submani- 
folds of $\boldsymbol{C} P^{m}$ we can classify complete 1-type submanifolds of $\boldsymbol{R} P^{m}$ without assuming minimality, thus generalizing the above result of Chen. Namely, we can embed $\boldsymbol{R} P^{m}$ in $\boldsymbol{C} P^{m}$ as a totally real totally geodesic submanifold such that the Hopf fibrations

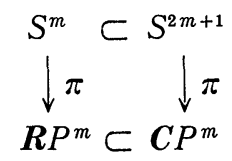

are compatible. Then a 1-type submanifold $M^{n} \subset \boldsymbol{R} P^{m}$ becomes a $C R$ (totally real) submanifold of $\boldsymbol{C} P^{m}$ and the $1^{\text {st }}$ standard embedding of $\boldsymbol{R} P^{m}$ is just the restriction of the $1^{\text {st }}$ standard embedding of $C P^{m}$. By Theorem 1 , case (2), it follows that $M^{n}$ is a totally real minimal submanifold of some totally geodesic complex $\boldsymbol{C} P^{n}$ in $\boldsymbol{C} P^{m}$. Therefore, $M^{n}$ is minimal in $\boldsymbol{C} P^{m}$ and since $\boldsymbol{R} P^{m} \subset \boldsymbol{C} P^{m}$ is totally geodesic it follows that $M^{n}$ is also minimal in $\boldsymbol{R} P^{m}$ so that the above result of Chen classifies $M^{n}$ as a totally geodesic $\boldsymbol{R} P^{n}$.

As above, for a compact submanifold $M^{n}$ of $\boldsymbol{R} P^{m}$ the first non-zero eigenvalue of the Laplacian satisfies

$$
\lambda_{1} \leqq 2(n+1)+\frac{n}{\operatorname{vol}(M)} \int_{M} \alpha^{2} d V
$$

with equality if and only if $M^{n}$ is the totally geodesic $\boldsymbol{R} P^{n}$.

Acknowledgements. The author would like to thank Professors D. Blair and B. Y. Chen for helpful discussions on the topic. In particular, the above classification of 1-type submanifolds of $\boldsymbol{R} P^{m}$ is based on a remark by Chen.

Added in Proof. It should be remarked that the classification results presented in this paper are essentially of local nature. Namely, Lemmas 2 and 3, Theorems 1 and 2 and Corollaries 1 and 2 are valid without assuming $M$ compact but merely complete. That is because Lemma 1, Theorem $\mathrm{A}$ and the result of [8] used in the proof are valid for any submanifold $M$.

\section{REFERENCES}

[1] Cecil, T., Geometric applications of critical point theory to submanifolds of complex projective space, Nagoya Math. J. 55 (1974), 5-31.

[2] Chen, B.Y., CR-Submanifolds of a Kaehler manifold, I, J. Differential Geometry 16 (1981) 305-322.

[3] Chen, B.Y., Total Mean Curvature and Submanifolds of Finite Type, World Scientific, Singapore 1984.

[4] Chen, B. Y. And Ogiue, K., On totally real submanifolds, Trans. Amer. Math. Soc. 193 (1974), 257-266.

[5] EjIRI, N., Calabi lifting and surface geometry in $S^{4}$, Tokyo J. Math. 9 (1986), 297-324. 
[6] Gauchman, H., Pinching theorems for totally real minimal submanifolds of $C P^{n}(c)$, Tohoku Math. J. 41 (1989), 249-257.

[7] Ludden, G., OKumura, M. And Yano, K., A totally real surface in $C P^{2}$ that is not totally geodesic, Proc. Amer. Math. Soc. 53 (1975), , 186-190.

[8] Martinez, A. AND Ros, A., On real hypersurfaces of finite type of $C P^{m}$, Kodai Math. J. 7 (1984) 304-316.

[9] NaitoH, H., Totally real parallel submanifolds in $P^{n}(C)$, Tokyo J. Math. 4 (1981), 279-306.

[10] Ros, A., Spectral geometry of $C R$-minimal submanifolds in the complex projective space, Kodai Math. J. 6 (1983) 88-99.

[11] Ros, A., On spectral geometry of Kaehler submanifolds, J. Math. Soc. Japan 36 (1984), 433-447.

[12] OpOzDA, B., On totally real surfaces with parallel mean curvature vector, Bull. Soc. Math. Belg. 40 (1988), 2, Ser B, 207-244.

[13] Sakamoto, K., Planar geodesic immersions, Tohoku Math. J. 29 (1977) 25-56.

[14] TAI, S.S., Minimum imbeddings of compact symmetric spaces of rank one, J. Differential Geometry, 2 (1968) 55-66.

[15] Takahashi, T., Minimal immersions of Riemannian manifolds, J. Math. Soc. Japan, 18 (1966) 380-385.

[16] URBano, F., Totally real minimal submanifolds of a complex projective space, Proc. Amer. Math. Soc. 93 (1985), 332-334.

Department of Mathematics

Penn State University/Fayette

P. O. Box 519

UNIONTOWN, PA 15401, USA 